\title{
Dysautonomia in early Parkinson disease: a window into the determinants of functional disability and an opportunity for early intervention
}

\author{
Aristide Merola ${ }^{1}$ - Elizabeth A. Coon ${ }^{2}$ \\ Received: 17 March 2020 / Accepted: 19 March 2020 / Published online: 2 May 2020 \\ (c) Springer-Verlag GmbH Germany, part of Springer Nature 2020
}

Keywords Parkinson disease $\cdot$ Orthostatic hypotension · Dysautonomia $\cdot$ Disability

In this issue of Clinical Autonomic Research, Sklerov and colleagues [1] examined the association between autonomic symptoms and activities of daily living (ADL) in the cohort of newly diagnosed patients enrolled in the Parkinson Progression Markers Initiative (PPMI) [2]. We welcome the addition of these results, which further increase the growing body of evidence indicating the critical relevance of autonomic dysfunction as a determinant of Parkinson disease (PD) functional disability [3].

As reported by the authors, autonomic dysfunction represents an early complication of PD, with a tendency to progress in prevalence and severity over the first 5 years since the diagnosis. These findings highlight the need for systematic screening with validated clinical tools, such as the SCOPA-AUT or the COMPASS-31, starting at the diagnosis of PD [4, 5].

The authors found an association between autonomic symptoms and ADL impairment, as well as depression, but not with motor symptoms. In particular, the cardiovascular subscore of the SCOPA-AUT, which assesses lightheadedness and presence of syncope, strongly predicted a decline in the ADL. These data highlight the critical role played by cardiovascular autonomic dysfunction, specifically symptoms related to orthostatic hypotension $(\mathrm{OH})$, on PD-associated functional disability, and reinforces the notion that early treatment of $\mathrm{OH}$ might improve PD functional outcomes. In previous studies, we found that, regardless of its

Aristide Merola

Aristide.Merola@osumc.edu

1 Department of Neurology, The Ohio State University Wexner Medical Center, Columbus, OH, USA

2 Department of Neurology, Mayo Clinic, Rochester, MN, USA symptomatic status, $\mathrm{OH}$ may result in significant impairment of gait and postural instability, as well as higher health-care utilization [6]. Still, the extent to which an aggressive treatment of $\mathrm{OH}$ might improve ADL and depression remains unclear.

The authors employed a mediation model to clarify whether the severity of motor symptoms contributed to the ADL disability, and found no associations between the impairment caused by autonomic symptoms and motor symptoms severity. These data support the hypothesis that multiple patterns of neurodegeneration may exist in PD. While motor symptoms relate to the nigrostriatal pathway dopaminergic degeneration, autonomic symptoms, as well as depression, involve different catecholaminergic systems, which are located in separate anatomical regions. The variable involvement of these non-dopaminergic systems may significantly influence the trajectory of PD disability progression.

The role of depression was carefully examined by the authors, investigating whether the use of antidepressants might impact the ADL outcomes. Interestingly, there were no differences associated with the use of pharmacological medications, suggesting a direct correlation with the pathological processes itself, rather than with the functional and social consequences associated with depression. Also, each clinical subdomain of the SCOPA-AUT was investigated separately, not finding associations of ADL outcomes with the thermoregulatory, pupillomotor, or sexual sub-scores of the scale. Whether this finding reflects a lower impact of these autonomic domains on the ADL, or a lack of statistical power due to co-segregation with the cardiovascular autonomic domain, or differential pathological processes, possibly involving distinct autonomic areas at separate stages of the disease, remains to be clarified. 
No sex differences were observed in this study. The cohort was $73 \%$ male, consistent with an increased prevalence of PD in males. However, there are specific sex differences that influence the presentation of PD [7]. Women are more likely to exhibit depression, postural instability, and difficulty in ADLs $[7,8]$. As these factors relate to the outcomes of this longitudinal study, the impact of sex is essential to evaluate. Additional limitations include the lack of evaluation of sleep disorders, which are known to be associated with dysautonomia and share some of the anatomical pathways involved in the pathophysiology of autonomic dysfunctions [9, 10]. Also, the ADL assessment was limited to the use of the Schwab and England (S\&E) scale only. While widely validated in $\mathrm{PD}$, the $\mathrm{S} \& \mathrm{E}$ is a relatively rudimental tool for the assessment of daily living activities, based on a single-item assessment. A more articulated clinical scale of ADL would have been useful to differentiate selected areas of impairment, potentially susceptible to specific therapeutic interventions.

In summary, the longitudinal approach to evaluating autonomic symptoms, particularly those relating to orthostatic hypotension, on activities of daily living found relevant results and led to further questions. Specifically, can early treatment of autonomic symptoms improve functional disability in patients with PD?

Funding None.

\section{Compliance with ethical standards}

Conflict of interest AM has received grant support from Lundbeck, advisory board compensation from Lundbeck, and speaker honoraria from Theravance Biopharma. EC has nothing to disclose.

\section{References}

1. Sklerov M, Shih CH, Browner N, Palma JA, Styner M, Dayan E (2020) Longitudinal change in autonomic symptoms predicts activities of daily living and depression in Parkinson's disease. Clin Auton Res. https://doi.org/10.1007/s10286-020-00672 -7[published online ahead of print, $2020 \mathrm{Feb} 20]$

2. Parkinson Progression Marker Initiative (2011) The Parkinson progression marker initiative (PPMI). Prog Neurobiol 95:629635. https://doi.org/10.1016/j.pneurobio.2011.09.005

3. Merola A, Romagnolo A, Rosso M et al (2018) Autonomic dysfunction in Parkinson's disease: a prospective cohort study. Mov Disord 33:391-397. https://doi.org/10.1002/mds.27268

4. Visser M, Marinus J, Stiggelbout AM, Van Hilten JJ (2004) Assessment of autonomic dysfunction in Parkinson's disease: the SCOPA-AUT. Mov Disord 19:1306-1312. https://doi. org/10.1002/mds. 20153

5. Sletten DM, Suarez GA, Low PA, Mandrekar J, Singer W (2012) COMPASS 31: a refined and abbreviated Composite Autonomic Symptom Score. Mayo Clin Proc 87:1196-1201. https://doi. org/10.1016/j.mayocp.2012.10.013

6. Merola A, Sawyer RP, Artusi CA et al (2018) Orthostatic hypotension in Parkinson disease: Impact on health care utilization. Parkinsonism Relat Disord 47:45-49. https://doi.org/10.1016/j. parkreldis.2017.11.344

7. Haaxma CA, Bloem BR, Borm GF et al (2007) Gender differences in Parkinson's disease. J Neurol Neurosurg Psychiatry 78:819824. https://doi.org/10.1136/jnnp.2006.103788

8. Perrin AJ, Nosova E, Co K et al (2017) Gender differences in Parkinson's disease depression. Parkinsonism Relat Disord 36:93-97. https://doi.org/10.1016/j.parkreldis.2016.12.026

9. Pilotto A, Romagnolo A, Tuazon JA et al (2019) Orthostatic hypotension and REM sleep behaviour disorder: impact on clinical outcomes in $\alpha$-synucleinopathies. J Neurol Neurosurg Psychiatry 90:1257-1263. https://doi.org/10.1136/jnnp-2019-320846

10. Coon EA, Cutsforth-Gregory JK, Benarroch EE (2018) Neuropathology of autonomic dysfunction in synucleinopathies. Mov Disord 33:349-358. https://doi.org/10.1002/mds.27186 